Proceedings of the XXIII Conference on Applied Crystallography, Krynica Zdrój, Poland, September 20-24, 2015

\title{
Metals and Polymers under Mechanical Stress: X-Ray Texture Analysis with the Use of a Tensile Stage
}

\author{
J. TE NiJenhuis*, N. Dadivanyan And D.J. GÖtz \\ PANalytical B.V., Almelo, The Netherlands
}

\begin{abstract}
X-ray diffraction studies have been performed to investigate the change of the preferred orientation of the crystallites as a function of externally applied mechanical stresses. For this purpose, a uniaxial tensile stage was integrated onto a Eulerian cradle in a laboratory X-ray diffraction system. Measurements have been executed while the sample experiences a certain mechanical loading. As example, orientation studies have been performed on metallic as well as on polymer samples.
\end{abstract}

DOI: 10.12693/APhysPolA.130.894

PACS/topics: 61.05.cp, 81.05.Bx, 81.05.Lg, 81.40.-Z

\section{Introduction}

In situ tensile testing is raising increasing interest in the world of materials science. By means of such experiments one can both investigate the properties of wellknown materials, and study the structure of newly created ones. Stress-strain experiments on metals and polymer foils or fibers often provide a better understanding of the structure-property relation.

In texture evolution studies, samples are often prepared ex situ and then mounted onto an X-ray diffraction system in order to measure the pole figures for a quantitative analysis. In the present study we have integrated a uniaxial tensile stage onto a Eulerian cradle in a laboratory X-ray diffraction system. The setup allows for performing in situ orientation studies as a result of the applied mechanical loading. The relationship between the stress and the amount of deformation reveals the elastic and plastic characteristics of the material. As an example, investigations have been performed on metallic as well as on polymeric samples.

\section{Experimental}

Mechanical deformation studies have been performed with the use of Deben 300N and Anton Paar TS 600 tensile stages, mounted onto a Eulerian three-axes cradle in a PANalytical Empyrean X-ray diffraction system. Samples are clamped with a pair of jaws and supported on stainless steel sliding bearings. The applied stress or strain are computer controlled. Extension rates can be set between $0.1 \mathrm{~mm} / \mathrm{min}$ and $1.5 \mathrm{~mm} / \mathrm{min}$.

The X-ray diffraction measurements were performed in parallel beam geometry with an X-ray lens in the incident beam path, and a parallel plate collimator with an equatorial acceptance angle of $0.27^{\circ}$ and a sealed proportional point detector in the diffracted beam path. The absence

\footnotetext{
*corresponding author; e-mail: hans.te.nijenhuis@panalytical.com
}

of defocusing effects over a large tilt range in this geometry greatly improves the quality of the orientation data. The polymer samples were measured using a pencil-like beam and an open 2D detector. As incident beam optic for reflection geometry an X-ray lens was used, whereas for transmission experiments a $2 \mathrm{D}$ focusing mirror of Kirkpatrick-Baez type was implemented, as described by Hooshmand et al. [1].

Pole figures were measured in reflection geometry, according to the Schulz reflection technique [2] with complete circles over the rotation angle $\phi\left(0^{\circ}-360^{\circ}\right)$ and fixed tilt angles $\chi$ over a range between $0^{\circ}$ and $75^{\circ}$. The $\phi$ rotation integration interval and the step size of the $\chi$ tilt-angle were $3^{\circ}$. A full representation of the texture over all crystal directions is given by the orientation distribution function (ODF), which was calculated using the Williams-Imhof-Matthies-Vinel (WIMV) method [3].

Thin strips of rolled copper $(25 \mathrm{~mm}$ length $\times 15 \mathrm{~mm}$ width $\times 0.02 \mathrm{~mm}$ thickness) were analyzed with increasing stresses up to $10 \mathrm{MPa}$. The sample was clamped in the tensile stage with the rolling direction (rotation angle $\phi=0^{\circ}$ ) parallel to the direction of the tensile stress. Pole figures of the $\{220\},\{222\},\{311\}$, and $\{400\}$ reflections of the face-centered cubic (fcc) structure of copper were measured on the sample in the initial status (extension $0 \mathrm{~mm}$ ) and subsequently at eight different extensions up to $7 \mathrm{~mm}$. During the pole figure measurements the elongation was kept constant for about four and a half hours.

The elongation rate was set to $0.2 \mathrm{~mm} / \mathrm{min}$ for the first four measurements and then increased to $1.0 \mathrm{~mm} / \mathrm{min}$ for the other measurements.

Polymer samples were measured both in reflection and transmission geometry. Correspondingly partial or complete Debye-Scherrer rings could be recorded. Based on $2 \mathrm{D}$ data, the orientation distribution as a function of strain can be revealed. The information obtained can be used for comparison with measurements of other mechanical and physical materials properties [4]. In case of reflection measurements the Deben 300N tensile stage was used. A thin rectangular strip of polyethylene 
(PE, $10 \mathrm{~mm}$ length $\times 11 \mathrm{~mm}$ width $\times 0.3 \mathrm{~mm}$ thickness $)$ was measured with increasing strain $\varepsilon$, where $\varepsilon=\Delta L / L_{0}$ with $\Delta L=L-L_{0}$ (the change in length of the sample) and $L_{0}$ is the original length of the sample. The strain has been gradually changed up to the value $\varepsilon=0.7$. The measurement spot on the sample was sufficiently small such that the strain could be assumed to be homogeneous. The $2 \mathrm{D}$ diffraction patterns were recorded fast (measurement time $1 \mathrm{~min}$ ) in the static mode of the PIXcel $^{3 \mathrm{D}} 2 \times 2$ detector (reflection geometry). The $2 \theta$ range $\left(17^{\circ}-28^{\circ}\right)$ was chosen such that the $\{110\}$ and $\{200\}$ reflections of the orthorhombic structure of PE could be observed. During each measurement the elongation was kept constant. The elongation rate was set to $1.5 \mathrm{~mm} / \mathrm{min}$ for all measurements. For transmission measurements a different tensile stage (Anton Paar TS 600) has been installed in the Empyrean diffractometer. Also in this case a thin polyethylene foil (PE, $10 \mathrm{~mm}$ length $\times 11 \mathrm{~mm}$ width $\times 0.3 \mathrm{~mm}$ thickness) was measured with increasing strain $\varepsilon$ up to $\varepsilon=0.7$. The complete $2 \mathrm{D}$ diffraction patterns were recorded (measurement time $15 \mathrm{~min}$ ) in the static mode of the PIXcel $^{3 \mathrm{D}}$ $2 \times 2$ detector (transmission geometry)

\section{Texture studies on rolled copper}

In texture analysis of rolled fcc metals, the ODF is often represented by a set of specific orientations (texture components) [5] as shown in Fig. 1 and Table I. These components are connected by fibers, where a fiber is a path of orientations through the ODF space. Most common fibers in fcc metals are the $\beta$ - and $\alpha$-fibers $[5,6]$.

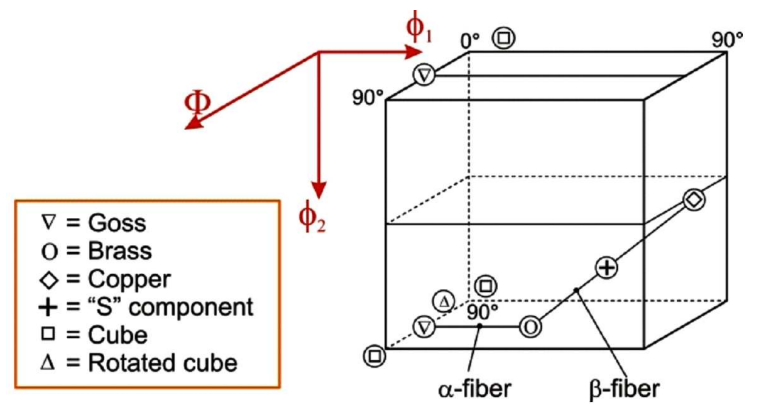

Fig. 1. Overview of fibers and components in ODF orientation space (after Rollett and Wright [5]).

Texture components in rolled fcc metals [5].

TABLE I

\begin{tabular}{c|c|c}
\hline \hline Name (symbol) & Miller indices & $\begin{array}{c}\text { Euler angles } \\
\text { Bunge }\left(\phi_{1}, \Phi, \phi_{2}\right)\end{array}$ \\
\hline copper $(\diamond)$ & $\{211\}\langle 111\rangle$ & $(90,35,45)$ \\
$\mathrm{S}(+)$ & $\{321\}\langle 436\rangle$ & $(35,65,35)$ \\
\hline brass $(\bigcirc)$ & $\{110\}\langle 112\rangle$ & $(35,45,90)$ \\
goss $(\nabla)$ & $\{110\}\langle 001\rangle$ & $(0,45,90)$ \\
\hline cube $(\square)$ & $\{100\}\langle 001\rangle$ & $(0,0,0)$ \\
rotated cube $(\Delta)$ & $\{520\}\langle 001\rangle$ & $(0,25,0)$
\end{tabular}

Figure 2 shows the force required to extend the copper strip as a function of the elongation. After an initial slight increase of the force in the lower extension range up to $c a .1 .5 \mathrm{~mm}$ the force flattens and steeply decreases above $2 \mathrm{~mm}$ until it reaches a steady state above $4 \mathrm{~mm}$.

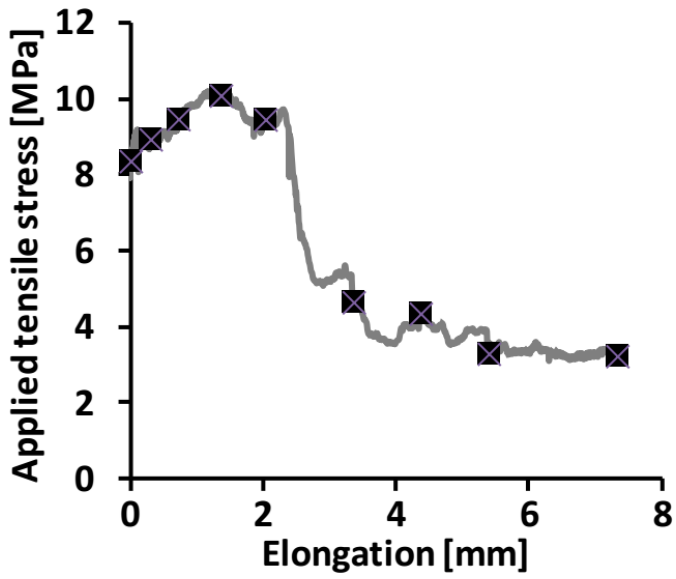

Fig. 2. Applied tensile stress as a function of the elongation of the sample. The squares on the curve indicate the extensions at which the pole figures were measured.

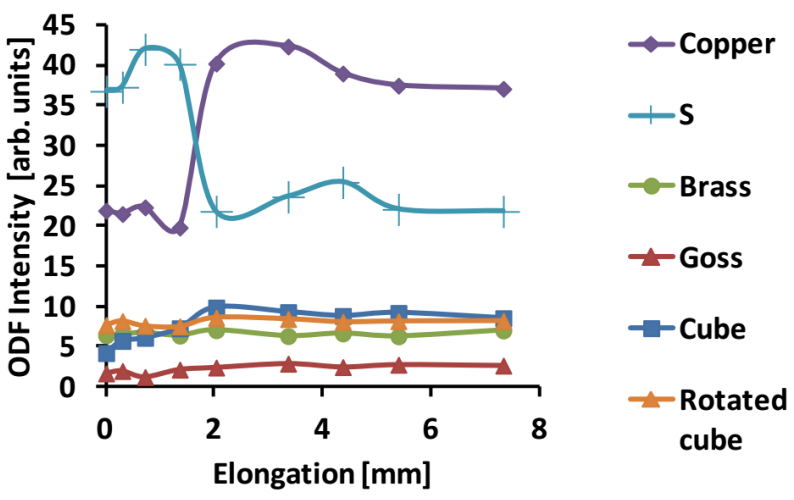

Fig. 3. Intensity of the main texture components as a function of the elongation of the sample.

Figure 3 shows the ODF intensity of the main components in fcc metals for the rolled copper strips at the various stages of deformation [7]. The intensity of most of the components is more or less independent of the elongation with two exceptions: the copper and $\mathrm{S}$ components show a strong change in intensity. Whereas the copper component strongly increases at an extension between $1.4 \mathrm{~mm}$ and $2.0 \mathrm{~mm}$, the $\mathrm{S}$ component strongly decreases in the same interval. After these transitions the intensities remain constant. The position of the intensity crossover coincides more or less with the steep decrease in force in the elongation curve in Fig. 2. Less clear but still visible is the slight increase of the cube component.

The initially strong $\beta$-fiber texture in the rolled copper specimen exhibited is in accordance with literature $[5,8]$. The intensity distribution along the fiber is not flat, but shows maximums of S-components at the first stages of the deformation process [7]. Copper is known to show a $\{111\}$ (copper-related) and a $\{100\}$ (cube-related) double 
fiber texture when it is placed under tensile stress [9]. The ratio of these two components depends on the initial texture of the sample. In our case of rolled copper, it is clearly demonstrated that the initial S-components move towards the copper component.

\section{Orientation studies on polymers}

When analyzing orientation of polymer materials (foils or fibers) a less complicated approach is followed. By analyzing 2D diffractograms one can obtain information on the orientation of the polymer chains. If a homogeneous distribution of intensity along the Debye-Scherrer rings ( $\gamma$-direction) is observed, a random orientation of the polymer chains can be assumed. In case a certain unior biaxial stretching was applied during manufacturing, a change in intensity distribution along the $\gamma$-direction is observed in the 2D diffraction patterns (Figs. 4 and 5). The intensity distribution is not homogeneous anymore, but exhibits maxima at specific angles. In the case of the polyethylene sample (Fig. 4) only a fraction of the Debye-Scherrer ring was monitored. Still, two effects can be clearly observed. Firstly, the relative intensity of the $\{200\}$ reflection increases with respect to the strongest $\{110\}$ reflection (Fig. 4c). Secondly, the intensity distribution, that was homogeneous in the initial state, shows a clear maximum in the $\gamma$-direction upon stretching (Fig. 4b).

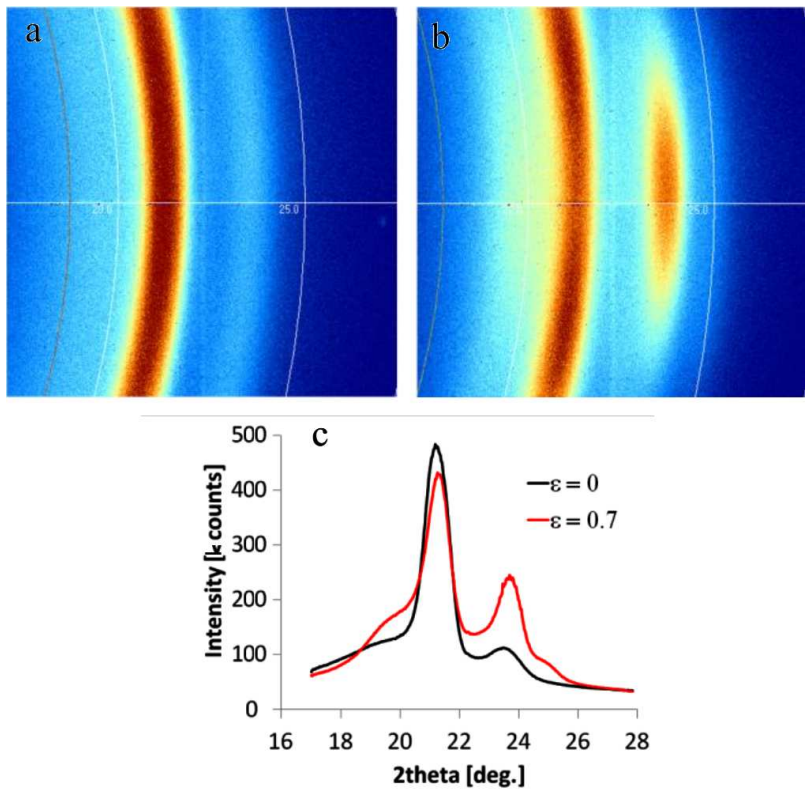

Fig. 4. 2D diffractograms of the PE sample in: (a) initial $(\varepsilon=0)$, (b) stretched $(\varepsilon=0.7)$ state, and (c) corresponding one-dimensional integration in the $2 \theta$ direction. The two peaks observed correspond to the $\{110\}$ and the $\{002\}$ reflections.

Complete Debye-Scherrer rings can be observed, if the $2 \mathrm{D}$ patterns are measured in transmission mode. It can be clearly seen in Fig. 5a,b that applying a strain on the $\mathrm{PE}$ sample results in reorientation of polymer chains. This effect can be nicely demonstrated when comparing
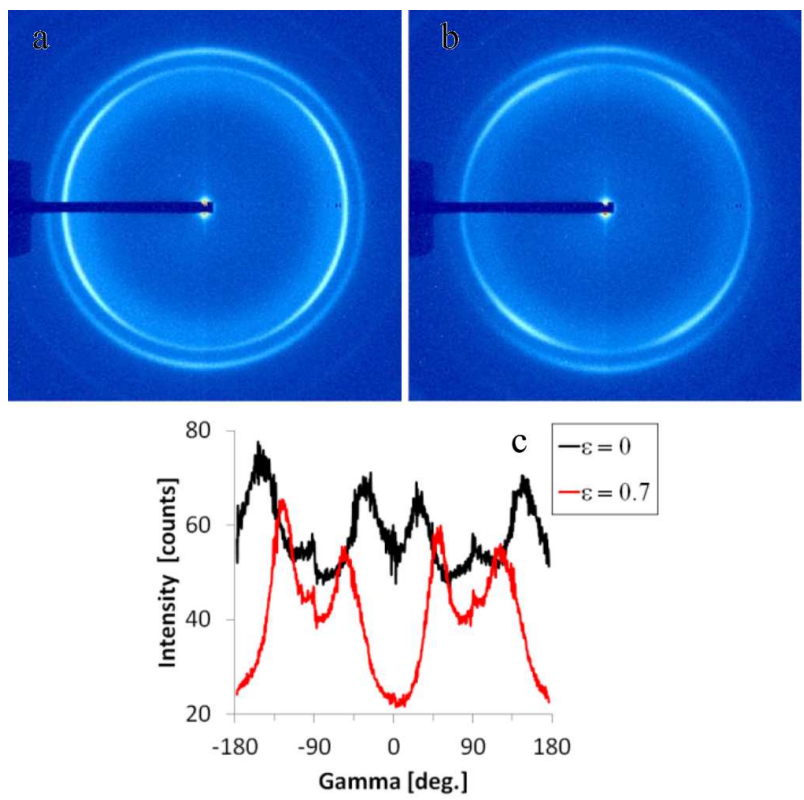

Fig. 5. 2D diffractograms of the PE sample in: (a) initial $(\varepsilon=0)$, (b) stretched $(\varepsilon=0.7)$ state, and (c) corresponding $1 \mathrm{D}$ integration in $\gamma$-direction.

the curves obtained from integration of the $\{110\}$ reflection $\left(21.6^{\circ} 2 \theta\right)$ in the $\gamma$-direction (Fig. 5c). In the measurement of the unstretched sample two minima of the intensity can be observed (at $\gamma= \pm 90^{\circ}$ ). When the sample is stretched to $\varepsilon=0.7$, four intensity minima can be detected (additional minima at $0^{\circ}$ and $180^{\circ}$ ). The intensity distribution of the second reflection at $\approx 24^{\circ} 2 \theta$ has changed upon stretching as well. The maxima at $\pm 90^{\circ}$ have become more pronounced whereas for the original sample amorphous halo was observed with only slight increase of intensity at $\gamma= \pm 90^{\circ}$.

\section{Conclusions}

We have shown the possibility of measuring complete pole figures using X-ray diffraction under the application of external tensile stresses. ODF analysis of measurements on a thin rolled copper strip with various extensions showed a change from the initial main component $\mathrm{S}$ to copper along the $\beta$-fiber.

Also the possibility of measuring partial as well as complete Debye-Scherrer rings both in reflection and transmission configuration of diffractometer on polymers has been demonstrated. These patterns have been performed qualitatively only thus far. The quality of the results shows that quantitative analysis in future research is well possible.

\section{Acknowledgments}

Dr. Gary Edwards, Deben UK Ltd., Woolpit, United Kingdom and Dr. Petra Kotnik, Anton Paar GmbH, Austria are gratefully acknowledged for providing the tensile stages used in this study. 


\section{References}

[1] S. Hooshmand, Y. Aitomäki, N. Norberg, A.P. Mathew, K. Oksman, ACS Appl. Mater. Interfaces 7, 13022 (2015).

[2] L.G. Schulz, J. Appl. Phys. 20, 1030 (1949).

[3] S. Matthies, G.W. Vinel, Phys. Status Solidi B 112, K111 (1982).

[4] R.L. Andersson, V. Ström, U.W. Gedde, P.E. Mallon, M.S. Hedenqvist, R.T. Olsson, Sci. Rep. 4, 6335 (2014).

[5] A.D. Rollett, S.I. Wright, in: Texture and Anisotropy, Eds. U.F. Kocks, C.N. Tomé, H.-R. Wenk, Cambridge University Press, Cambridge, UK 1998, Ch. 5.
[6] S.-H. Kim, S.Z. Han, C.J. Kim, I.-Y. Hwang, F. Yin, Mater. Trans. 50, 537 (2009).

[7] J. te Nijenhuis, N. Dadivanyan, D.J. Götz, Mater. Res. Soc. Symp. Proc. 1754, 123 (2015).

[8] J. Hirsch, K. Lücke, Acta Metall. 36, 2863 (1988).

[9] O. Engler, V. Randle, Introduction to Texture Analysis: Macrotexture, Microtexture, and Orientation Mapping, 2nd ed., CRC Press, Boca Raton 2009. 